\title{
THEORY OF MODELING AND SIMULATION
}

\author{
by Bernard P. Zeigler, Herbert Praehofer, Tag Gon Kim \\ 2nd Edition, Academic Press, 2000, ISBN: 0127784551
}

\begin{abstract}
$\mathrm{G}$ iven the many advances in modeling and simulation in the last decades, the need for a widely accepted framework and theoretical foundation is becoming increasingly necessary. Methods of modeling and simulation are fragmented across disciplines making it difficult to re-use ideas from other disciplines and work collaboratively in multidisciplinary teams. Model building and simulation is becoming easier and faster through implementation of advances in software and hardware. However, difficult and fundamental issues such as model credibility and interoperation have received less attention. These issues are now addressed under the impetus of the High Level Architecture (HLA) standard mandated by the U.S. DoD for all contractors and agencies.
\end{abstract}

This book concentrates on integrating the continuous and discrete paradigms for modeling and simulation. A second major theme is that of distributed simulation and its potential to support the co-existence of multiple formalisms in multiple model components. Prominent throughout are the fundamental concepts of modular and hierarchical model composition. These key ideas underlie a sound methodology for construction of complex system models.

The book presents a rigorous mathematical foundation for modeling and simulation. It provides a comprehensive framework for integrating various simulation approaches employed in practice, including such popular modeling methods as cellular automata, chaotic systems, hierarchical block diagrams, and Petri Nets. A unifying concept, called the DEVS Bus, enables models to be transparently mapped into the Discrete Event System Specification (DEVS). The book shows how to construct computationally efficient, object-oriented simulations of DEVS models on parallel and distributed environments. In designing integrative simulations, whether or not they are HLA compliant, this book provides the foundation to understand, simplify and successfully accomplish the task. 


\title{
Library Update
}

\section{MODELING HUMAN AND ORGANIZATIONAL BEHAVIOR: APPLICATION TO MILITARY SIMULATIONS}

\author{
Editors: Anne S. Mavor, Richard W. Pew \\ National Academy Press, 1999, ISBN: 0309060966. Hardcover - 432 pages.
}

$\mathrm{T}$ his book presents a comprehensive treatment of the role of the human and the organization in military simulations. The issue of representing human behavior is treated from the perspective of the psychological and organizational sciences. After a thorough examination of the current military models, simulations and requirements, the book focuses on integrative architectures for modeling the individual combatant, followed by separate chapters on attention and multitasking, memory and learning, human decision making in the framework of utility theory, models of situation awareness and enabling technologies for their implementation, the role of planning in tactical decision making, and the issue of modeling internal and external moderators of human behavior.

The focus of the tenth chapter is on modeling of behavior at the unit level, examining prior work, organizational unit-level modeling, languages and frameworks. It is followed by a chapter on information warfare, discussing models of information diffusion, models of belief formation and the role of communications technology. The final chapters consider the need for situation-specific modeling, prescribe a methodology and a framework for developing human behavior representations, and provide recommendations for infrastructure and information exchange.

The book is a valuable reference for simulation designers and system engineers. 


\title{
Library Update
}

\section{HANDBOOK OF SIMULATOR-BASED TRAINING}

\author{
by Eric Farmer (Ed.), Johan Reimersma, Jan Moraal, Peter Jorna \\ Ashgate Publishing Company, 1999, ISBN: 0754611876.
}

$\mathrm{T}$ he rapidly expanding area of military modeling and simulation supports decision making and planning, design of systems, weapons and infrastructure. This particular book treats the third most important area of modeling and simulation training. It starts with thorough analysis of training needs, covering mission analysis, task analysis, trainee and training analysis. The second section of the book treats the issue of training program design, examining current practices, principles of training and instruction, sequencing of training objectives, specification of training activities and scenarios, methodology of design and optimization of training programs. In the third section the authors introduce the problem of training media specification and treat technical issues such as databases and models, human-simulator interfaces, visual cueing and image systems, haptic, kinaesthetic and vestibular cueing, and finally, the methodology for training media specification. The final section of the book is devoted to training evaluation, covering the topics of performance measurement, workload measurement, and team performance. In the concluding part the authors outline the trends in using simulators for training.

The primary audience for this book is the community of managers and experts involved in training operators. It can also serve as useful reference for designers of training simulators. 


\title{
CREATING COMPUTER SIMULATION SYSTEMS: An Introduction to the High Level Architecture
}

\author{
by Frederick Kuhl, Richard Weatherly, Judith Dahmann \\ Prentice Hall, 1999, ISBN: 0130225118. - 212 pages.
}

\begin{abstract}
$\mathrm{G}$ iven the increasing importance of simulations in nearly all aspects of life, the authors find that combining existing systems is much more efficient than building newer, more complex replacements. Whether the interest is in business, the military, or entertainment or is even more general, the book shows how to use the new standard for building and integrating modular simulation components and systems. The HLA, adopted by the U.S. Department of Defense, has been years in the making and recently came ahead of its competitors to grab the attention of engineers and designers worldwide.

The book and the accompanying CD-ROM set contain an overview of the rationale and development of the HLA; a Windows-compatible implementation of the HLA Runtime Infrastructure (including test software). It allows the reader to understand indepth the reasons for the definition of the HLA and its development, how it came to be, how the HLA has been promoted as an architecture, and why it has succeeded. Of course, it provides an overview of the HLA examining it as a software architecture, its large pieces, and chief functions; an extended, integrated tutorial that demonstrates its power and applicability to real-world problems; advanced topics and exercises; and well-thought-out programming examples in text and on disk.
\end{abstract}

The book is well-indexed and may serve as a guide for managers, technicians, programmers, and anyone else working on building simulations. 


\title{
HANDBOOK OF SIMULATION: \\ Principles, Methodology, Advances, Applications, and Practice
}

\author{
edited by Jerry Banks \\ John Wiley \& Sons, 1998, ISBN: 0471134031. Hardcover - 864 pages.
}

$\mathrm{S}$ imulation modeling is one of the most powerful techniques available for studying

large and complex systems. This book is the first ever to bring together the top 30 international experts on simulation from both industry and academia. All aspects of simulation are covered, as well as the latest simulation techniques. Most importantly, the book walks the reader through the various industries that use simulation and explains what is used, how it is used, and why.

This book provides a reference to important topics in simulation of discrete- event systems. Contributors come from academia, industry, and software development. Material is arranged in sections on principles, methodology, recent advances, application areas, and the practice of simulation. Topics include object-oriented simulation, software for simulation, simulation modeling, and experimental design. For readers with good background in calculus based statistics, this is a good reference book.

Applications explored are in fields such as transportation, healthcare, and the military. Includes guidelines for project management, as well as a list of software vendors. The book is co-published by Engineering and Management Press. 


\title{
ADVANCES IN MISSILE GUIDANCE THEORY
}

\author{
by Joseph Z. Ben-Asher, Isaac Yaesh \\ AIAA, 1998, ISBN 1-56347-275-9.
}

$\mathrm{T}$

his book about terminal guidance of intercepting missiles is oriented toward practicing engineers and engineering students. It contains a variety of newly developed guidance methods based on linear quadratic optimization problems. This application-oriented book applies widely used and thoroughly developed theories such LQ and H-infinity to missile guidance. The main theme is to systematically analyze guidance problems with increasing complexity. Numerous examples help the reader to gain greater understanding of the relative merits and shortcomings of the various methods. Both the analytical derivations and the numerical computations of the examples are carried out with MATLAB Companion Software: The authors have developed a set of MATLAB M-files that are available on a diskette bound into the book.

\section{CONTROL OF SPACECRAFT AND AIRCRAFT}

\author{
by Arthur E. Bryson, Jr. \\ Princeton University Press, 1994, ISBN 0-691-08782-2.
}

\footnotetext{
This text provides an overview and summary of flight control, focusing on the best 1 possible control of spacecraft and aircraft, i.e., the limits of control. The minimum output error responses of controlled vehicles to specified initial conditions, output commands, and disturbances are determined with specified limits on control authority. These are determined using the linear-quadratic regulator (LQR) method of feedback control synthesis with full-state feedback. An emphasis on modeling is also included for the design of control systems. The book includes a set of MATLAB Mfiles in companion software
} 